\title{
Goldstones in diphotons
}

\author{
Brando Bellazzini, ${ }^{a, b}$ Roberto Franceschini, ${ }^{c}$ Filippo Sala $^{d}$ and Javi Serra ${ }^{c}$ \\ ${ }^{a}$ Institut de Physique Théorique, Université Paris Saclay, \\ CEA, CNRS, F-91191 Gif-sur-Yvette, France \\ ${ }^{b}$ Dipartimento di Fisica e Astronomia, Università di Padova, \\ Via Marzolo 8, I-35131 Padova, Italy \\ ${ }^{c}$ CERN, Theory Division, \\ Geneva, Switzerland \\ ${ }^{d}$ LPTHE, CNRS, UMR 7589, \\ 4 Place Jussieu, F-75252, Paris, France \\ E-mail: brando.bellazzini@cea.fr, roberto.franceschini@cern.ch, \\ fsala@lpthe.jussieu.fr, javier.serra.mari@cern.ch
}

ABSTRACT: We study the conditions for a new scalar resonance to be observed first in diphotons at the LHC Run-2. We focus on scenarios where the scalar arises either from an internal or spacetime symmetry broken spontaneously, for which the mass is naturally below the cutoff and the low-energy interactions are fixed by the couplings to the broken currents, UV anomalies, and selection rules. We discuss the recent excess in diphoton resonance searches observed by ATLAS and CMS at $750 \mathrm{GeV}$, and explore its compatibility with other searches at Run-1 and its interpretation as Goldstone bosons in supersymmetry and composite Higgs models. We show that two candidates naturally emerge: a Goldstone boson from an internal symmetry with electromagnetic anomalies, and the scalar partner of the Goldstone of supersymmetry breaking: the sgoldstino. The dilaton from conformal symmetry breaking is instead disfavoured by present data, in its minimal natural realization.

KEywords: Beyond Standard Model, Supersymmetric Standard Model, Technicolor and Composite Models

ARXIV EPRINT: 1512.05330 


\section{Contents}

1 Introduction 1

2 Pseudo-Goldstone boson 5

$\begin{array}{llr}3 & \text { Sgoldstino } & 9\end{array}$

4 Dilaton 13

5 Conclusions $\quad 15$

\section{Introduction}

After the discovery of a light Higgs at the LHC Run-1, experimental efforts have shifted towards the search for new scalars beyond the SM. Evidence of another spin-0 resonance would be indicative of a natural solution of the electroweak hierarchy problem, as opposed to anthropic arguments, given that elementary scalars are generically unnatural in quantum field theory. As a matter of fact, extra scalars are common in both the two prototype solutions of the hierarchy problem, supersymmetry (SUSY) and composite Higgs models. Their experimental signatures at colliders are however model dependent, since they depend on the different selection rules of the specific realizations. An interesting question which we wish to address in this work is what are the conditions for the leading signal to be found in the diphoton channel $p p \rightarrow \Phi \rightarrow \gamma \gamma$, consistently with null resonance searches in other experimental analyses. We are motivated by the anomaly [1-3] observed at the LHC by the ATLAS and CMS collaborations, which we take as a benchmark for a new scalar resonance $\Phi$ glimpsed for the first time in the diphoton channel.

The anomaly is characterized by the signal strength, $\mu_{13}^{\gamma \gamma}=\sigma_{13 \mathrm{TeV}} \times \mathrm{BR}_{\gamma \gamma}$, mass and total width shown in table 1 . The difference between the observed and expected number of events in the bins of the excess is presently around 10. Therefore the statistical uncertainty on the excess signal strength is, at best, a few $\times 10 \%$. Given the small number of signal events, it is premature to rely on any indication of a large resonance width. In fact, ATLAS reported a large local significance for both the narrow width and large width line-shapes used to characterize the excess. We therefore treat the preferred width quoted by ATLAS [2] as an upper bound. Despite the small number of excess events, the relatively small amount of expected background events, and the bounds from Run-1 searches in the same final state, make it reasonable to speculate that such a signal is indeed the first glimpse of BSM physics at the LHC.

A priori either a spin-0 or spin-2 boson, neutral under electromagnetism and colour, could reproduce such an excess over the SM expectation. Although with a poor significance, 


\begin{tabular}{|c|c|c|}
\hline$\mu_{13}^{\gamma \gamma}(\mathrm{fb})$ & $m(\mathrm{GeV})$ & $\Gamma(\mathrm{GeV})$ \\
\hline 7 & 750 & $\leq 45$ \\
\hline
\end{tabular}

Table 1. Signal strength in diphotons, mass and width suggested by the ATLAS and CMS analyses.

\begin{tabular}{|c|c|c|c|c|c|c|c|c|c|c|}
\hline & $\frac{\sigma_{13 \mathrm{TeV}}}{\sigma_{8} \mathrm{TeV}}$ & $10^{2} \times$ & $r_{W W}^{\gamma}$ & $r_{Z Z}^{\gamma}$ & $r_{Z \gamma}^{\gamma}$ & $r_{h h}^{\gamma}$ & $r_{t \bar{t}}^{\gamma}$ & $r_{\tau \bar{\tau}}^{\gamma}$ & $r_{\ell \bar{\ell}}^{\gamma}$ & $r_{g g}^{\gamma}$ \\
\hline ATLAS & $2.9[5]^{*}$ & & $3.0[7]$ & $13[9]^{*}$ & $19[10]^{*}$ & $4.1[11]^{*}$ & $0.22[13]$ & $15[15]$ & $124[17]^{*}$ & 0.14 [19] \\
\hline CMS & $4.0[6]$ & & $0.5[8]$ & $4.6[8]$ & - & $2.8[12]^{*}$ & $0.33[14]$ & $7.4[16]$ & $114[18]^{*}$ & $0.083[20]^{*}$ \\
\hline
\end{tabular}

Table 2. Experimental lower bounds on the ratio of production cross sections at 13 and $8 \mathrm{TeV}$, and on $10^{2} \times r_{X Y}^{\gamma}$ from the $8 \mathrm{TeV}$ run of LHC, where $r_{X Y}^{\gamma} \equiv \Gamma_{\gamma \gamma} / \Gamma_{X Y}$. We dub with a star * the bounds where the width assumed in the corresponding experimental analysis is smaller than $45 \mathrm{GeV}$.

the observed width-over-mass ratio $\Gamma / m \approx 6 \%$ would indicate that some of the effective couplings $g_{\Phi}$ of $\Phi$ to lighter states, either in the SM or beyond, should be somewhat sizeable,

$$
\frac{\Gamma_{\Phi}}{m_{\Phi}} \sim\left(\frac{g_{\Phi}}{4 \pi}\right)^{2} \pi N \sim 6 \%\left(\frac{g_{\Phi}}{1}\right)^{2}\left(\frac{N}{3}\right),
$$

where $N$ is the multiplicity factor of the final state, including polarizations. We are defining the effective coupling $g_{\Phi}$ in a loose sense through the decay amplitude $\mathcal{M}_{1 \rightarrow 2} \sim g_{\Phi} m_{\Phi}$. For instance, the effective coupling of $\Phi$ in a derivative interaction such as $c_{H} \Phi / f\left|D_{\mu} H\right|^{2}$ is set by the energy squared as $g_{\Phi} \sim c_{H} E^{2} /\left(f m_{\Phi}\right)=c_{H} m_{\Phi} / f{ }^{1}$. The observed signal strength at LHC Run-2, along with the exclusion bound on diphotons at LHC Run-1, are reported in table 2 . They place a lower bound on the ratio of production cross sections $\sigma_{13 \mathrm{TeV}} / \sigma_{8 \mathrm{TeV}} \gtrsim$ 4.0 , that indicates that the dominant production mode of the new resonance could be gluon fusion $g g \rightarrow \Phi$, for which $\left(\sigma_{13 \mathrm{TeV}} / \sigma_{8 \mathrm{TeV}}\right)_{g g \rightarrow \Phi} \approx 4.7$ [4]. Hereafter we will consider resonances produced in this channel (and comment shortly on photon fusion). We will also restrict the discussion to the case of $\Phi$ being a spin- 0 resonance, and assume invariance under CP (motivated by the nonobservation of CP violation beyond the $\mathrm{SM}$ ); we refer in the following to $\Phi=\sigma$ and $\Phi=\eta$ for a CP-even and CP-odd scalar boson, respectively.

In table 2 we also show the lower bounds on the ratios of decay rates, $r_{X Y}^{\gamma} \equiv \Gamma_{\gamma \gamma} / \Gamma_{X Y}$, set by null searches at LHC Run-1 in the various channels other than the decay into diphotons. In deriving those constraints, we assumed that the production cross section scales with the $g g$ parton luminosity, using the MSTW2008 parton distribution functions [4] for definiteness. Strong bounds come from $8 \mathrm{TeV}$ resonance searches in leptons, $\tau$ pairs, and dijets. However, from a theoretical point of view, these constraints are not very severe or difficult to evade, as chiral symmetry controls the size of the Yukawa couplings of $\Phi$ through the fermion masses.

With this theoretical prior, we assume the following structure for the effective phenomenological lagrangian of $\Phi$ :

$$
\mathcal{L}=\mathcal{L}^{\mathrm{SM}}+\frac{1}{2}\left(\partial_{\mu} \Phi\right)^{2}-\frac{1}{2} m_{\Phi}^{2} \Phi^{2}+\mathcal{L}_{I}^{\Phi}, \quad \Phi=\sigma, \eta,
$$

\footnotetext{
${ }^{1}$ Notice that the conclusion extracted from eq. (1.1) relies on the assumption that a single resonance contributes to the excess.
} 
where

$$
\begin{aligned}
\mathcal{L}_{I}^{\Phi=\sigma}= & \frac{\sigma}{f}\left(c_{h} \partial_{\mu} h \partial^{\mu} h+c_{Z} m_{Z}^{2} Z_{\mu} Z^{\mu}+2 c_{W} m_{W}^{2} W_{\mu} W^{\mu}\right) \\
& -\frac{\sigma}{f}\left(c_{t} m_{t} \bar{t} t+c_{b} m_{b} \bar{b} b+c_{\tau} m_{\tau} \bar{\tau} \tau\right) \\
& +\frac{\sigma}{f}\left(c_{g g} \frac{\alpha_{3}}{8 \pi} G_{\mu \nu}^{2}+c_{\gamma \gamma} \frac{\alpha_{e}}{8 \pi} F_{\mu \nu}^{2}\right) \\
& +\frac{\sigma}{f}\left(c_{W W} \frac{\alpha_{2}}{4 \pi} W_{\mu \nu}^{+} W^{-\mu \nu}+c_{Z Z} \frac{\alpha_{2} \cos ^{2} \theta_{W}}{8 \pi} Z_{\mu \nu}^{2}+c_{Z \gamma} \frac{\alpha_{e}}{4 \pi \tan \theta_{W}} Z_{\mu \nu} F^{\mu \nu}\right) \\
& -\frac{\sigma}{f} c_{3} m_{h}^{2} h^{2}
\end{aligned}
$$

and

$$
\begin{aligned}
\mathcal{L}_{I}^{\Phi=\eta}= & -i \frac{\eta}{f}\left(C_{t} m_{t} \bar{t} \gamma^{5} t+C_{b} m_{b} \bar{b} \gamma^{5} b+C_{\tau} m_{\tau} \bar{\tau} \gamma^{5} \tau\right) \\
& -\frac{\eta}{f}\left(C_{g g} \frac{\alpha_{3}}{8 \pi} G_{\mu \nu}^{a} \tilde{G}^{a \mu \nu}+C_{\gamma \gamma} \frac{\alpha_{e}}{8 \pi} F_{\mu \nu} \tilde{F}_{\mu \nu}\right) \\
& -\frac{\eta}{f}\left(C_{W W} \frac{\alpha_{2}}{4 \pi} W_{\mu \nu}^{+} \tilde{W}^{-\mu \nu}+C_{Z Z} \frac{\alpha_{2} \cos ^{2} \theta_{W}}{8 \pi} Z_{\mu \nu} \tilde{Z}^{\mu \nu}+C_{Z \gamma} \frac{\alpha_{e}}{4 \pi \tan \theta_{W}} Z_{\mu \nu} \tilde{F}^{\mu \nu}\right),
\end{aligned}
$$

where $\tilde{F}^{\mu \nu} \equiv \epsilon^{\mu \nu \rho \sigma} F_{\rho \sigma}$ for any of the field strengths, and $\theta_{W}$ is the weak angle. We did not include terms with more than one $\Phi$-leg, given they are irrelevant for the phenomenology we discuss in this paper. In full analogy with the electroweak vacuum expectation value $v \simeq 246 \mathrm{GeV}$ or the pion decay constant in QCD $f_{\pi}$, the scale $f$ appearing in the effective lagrangians above does not represent in general a physical mass threshold. Instead the mass of the heavy BSM states associated with the scale $f$ are controlled by that scale times a coupling $m_{*}=g_{*} f$, like $m_{W}=g v / 2$ or $m_{\rho} \approx g_{\rho} f_{\pi}$. We did not introduce flavor indices, and we will be assuming no flavor violating couplings for $\Phi$. Notice that we parametrize the couplings to the gauge field strengths following the generic expectation in weakly coupled theories of a loop suppression $\alpha / 4 \pi$. However this expectation is challenged by the signal excess, which neglecting $K$ factors we find to be well approximated by

$$
\begin{aligned}
& \mu_{13, \sigma}^{\gamma \gamma} \simeq 7 \mathrm{fb}\left(\frac{\left|c_{g g}^{\mathrm{eff}} \| c_{\gamma \gamma}^{\mathrm{eff}}\right|}{500}\right)^{2}\left(\frac{500 \mathrm{GeV}}{f}\right)^{4}\left(\frac{45 \mathrm{GeV}}{\Gamma_{\sigma}}\right), \\
& \mu_{13, \eta}^{\gamma \gamma}=\mu_{13, \sigma}^{\gamma \gamma}\left(c_{g g, \gamma \gamma}^{\mathrm{eff}} \rightarrow 2 C_{g g, \gamma \gamma}^{\mathrm{eff}}\right),
\end{aligned}
$$

where $\left|c_{g g, \gamma \gamma}^{\text {eff }}\right|$ and $\left|C_{g g, \gamma \gamma}^{\text {eff }}\right|$ parametrize the effective coupling to $g g$ and $\gamma \gamma$ from the contact terms $c_{g g, \gamma \gamma}$, and $C_{g g, \gamma \gamma}$ respectively, as well as from loops of the SM fermions and $W$ 's. See eqs. (2.7) and (4.4) for the impact of the loops in $\left|C_{g g, \gamma \gamma}^{\text {eff }}\right|$ and $\left|c_{g g, \gamma \gamma}^{\text {eff }}\right|$ respectively. We advance that the requirement of large $\left|c_{\gamma \gamma}^{\text {eff }}\right|$ or $\left|C_{\gamma \gamma}^{\mathrm{eff}}\right|$, combined with the experimental constrains from $t \bar{t}$ and $V V, V=\{W, Z\}$, discussed below, implies that if no new physics contributions to the effective couplings of $\Phi$ to photons are present $\left(c_{\gamma \gamma}=C_{\gamma \gamma}=0\right)$, the excess cannot be reproduced.

Let us remark at this point our interpretation of the resonance $\Phi$ as a pseudo-Goldstone boson (pGB) of some kind, to be expanded in the sections below. The required sizable 
couplings of a generic scalar $\Phi$ to photons (gluons) can arise from loops of multiple electrically (colour) charged states, $X$, coupled to $\Phi$. Nevertheless, on fairly general grounds one expects these states to contribute to the mass of $\Phi$ at the same loop order,

$$
\left(\Delta m_{\Phi}^{2}\right)_{X} \sim N_{X} \frac{y_{X}^{2}}{16 \pi^{2}} m_{*}^{2}
$$

with $N_{X}$ their multiplicity and $y_{X}$ their coupling to $\Phi$. This suggests that in the absence of a protection mechanism or tuning, $\Phi$ would not be separated from the cutoff $m_{*}$ of our effective field theory, possibly compromising calculability and predictivity. This is just the familiar hierarchy problem that plagues any theory with unprotected scalars. This argument holds for weakly coupled theories as well as for strongly coupled ones where $\Phi$ arises as a composite state of the underlying dynamics. For the latter, even though $X$ particles might not be required to generate large couplings to photons (gluons), that is $c_{\gamma \gamma}\left(c_{g g}\right) \gg 1$, one expects $m_{\Phi} \ll m_{*}$ only by accident. Because of these reasons, an (approximate) shift-symmetry acting on $\Phi$ will be our rationale for its lightness compared to $m_{*}$. The same selection rules control also the $\Phi$ couplings that do not respect the shift symmetry (this will become apparent in the sections that follow).

Since we consider production via gluon fusion, a non-vanishing decay rate to gluons is constrained by $8 \mathrm{TeV}$ resonance searches in dijets,

$$
r_{g g}^{\gamma} \simeq \frac{1}{8}\left(\frac{\alpha_{e}}{\alpha_{3}}\right)^{2}\left(\frac{\left|c_{\gamma \gamma}^{\mathrm{eff}}\right|}{\left|c_{g g}^{\mathrm{eff}}\right|}\right)^{2},
$$

for both the scalar and pseudo-scalar $\left(c_{g g, \gamma \gamma}^{\mathrm{eff}} \rightarrow C_{g g, \gamma \gamma}^{\mathrm{eff}}\right)$ candidates.

Furthermore, given the interactions of the scalar $\sigma$, we can identify the most stringent constraints as those coming from $p p \rightarrow \Phi \rightarrow V V, h h$ where $V=\{W, Z\}$. The corresponding decay widths scale with $m_{\sigma}^{3}$, and the theoretical prediction for the ratio of decay rates reads

$$
\Phi=\sigma: \quad r_{V V}^{\gamma} \simeq n_{V}\left(\frac{\alpha_{e}}{4 \pi}\right)^{2}\left(\frac{c_{\gamma \gamma}}{c_{V}}\right)^{2}, \quad r_{h h}^{\gamma} \simeq 2\left(\frac{\alpha_{e}}{4 \pi}\right)^{2}\left(\frac{c_{\gamma \gamma}}{c_{h}}\right)^{2} .
$$

where $n_{Z}=1$ and $n_{W}=2$. Compatibility with the corresponding bounds shown in table 2 requires very large $c_{\gamma \gamma} / c_{h, V}=O\left(16 \pi^{2}\right)$. In these general estimates, just for simplicity of the exposition, we neglected $O\left(m_{V, h} / m_{\sigma}\right)$ and the top loop contribution to $\gamma \gamma$ (notice that the contribution to $\Gamma_{h h}$ from $c_{3}$ is generically suppressed by $m_{h}^{2} / m_{\sigma}^{2}$ ). We point out also that in models with custodial symmetry $c_{h}=c_{W}=c_{Z}$, and in that case the strongest constraint comes from $Z Z$ resonance searches at Run-1. Such large values of $c_{\gamma \gamma} / c_{h, V}$ can be obtained by suppressing $c_{h, V}$ and/or boosting $c_{\gamma \gamma}$, as it may happen when the couplings to $V V$, $h h$ and $\gamma \gamma$ are generated at the same loop order, i.e. either all at tree- or loop-level. In section 3 we discuss such a scenario where $\sigma$ is the sgoldstino, for which all the couplings can arise at tree-level, resulting in $c_{\gamma \gamma} / c_{h, V}=O\left(16 \pi^{2}\right)$. Alternatively, it may happen that while the coupling to photons is formally at one-loop and $c_{h, V}$ is tree-level, the former is endowed with an anomalously large factor like in the dilaton model we present in section 4, where $c_{\gamma \gamma}$ counts new degrees of freedom charged under electromagnetism. 
The CP-odd scalar $\eta$ instead has a built-in selection rule, $\mathrm{CP}$ invariance, that forbids the tree-level coupling to the longitudinal $V$, and $h$, so that the bounds from $r_{V V, h h}^{\gamma}$ are satisfied more easily. Instead for the pseudo-scalar the bounds from $t \bar{t}$ can become relevant, as $C_{t}$ respects CP. However, since the partial decay width into tops scales with $m_{\eta}$ (to be contrasted with the one in diphotons that goes like $m_{\eta}^{3}$ ), and the bound on $r_{t \bar{t}}^{\gamma}$ is milder, a slightly smaller coupling $C_{t}$ or an enhanced anomaly coefficient $C_{\gamma \gamma}=O($ few $)$ are enough to satisfy the experimental constraints:

$$
\Phi=\eta: \quad r_{t \bar{t}}^{\gamma} \simeq \frac{2}{3}\left(\frac{\alpha_{e}}{4 \pi}\right)^{2}\left(\frac{m_{\eta}}{m_{t}}\right)^{2}\left(\frac{\left|C_{\gamma \gamma}^{\mathrm{eff}}\right|}{C_{t}}\right)^{2}, \quad \frac{\left|C_{\gamma \gamma}^{\mathrm{eff}}\right|}{C_{t}}=\left|\frac{C_{\gamma \gamma}}{C_{t}}+\frac{2}{3} A_{1 / 2}\left(x_{t}\right)\right|,
$$

where $A_{1 / 2}\left(x_{t}\right), x_{t}=4 m_{t}^{2} / m_{\eta}^{2}$, is the contribution of the top loop to the effective $\gamma \gamma$ coupling. In the next section we discuss in detail this natural case in the context of a pGB emerging from an anomalous $\mathrm{U}(1)$ that is broken spontaneously.

In theories where the electroweak $\mathrm{SU}(2)_{L} \times \mathrm{U}(1)_{Y}$ symmetry is linearly realized above the scale $f>v$, such as the ones we consider in this paper, a non-trivial constraint arises from $Z \gamma$ or $Z Z$ resonance searches (for the latter even if $c_{Z}=0$ ). This is clear once the $\gamma \gamma$ coupling for $\Phi$ is written in a manifestly symmetric form

$$
\mathcal{L}_{I}^{\Phi=\eta} \supset-\frac{\eta}{f}\left(C_{W} \frac{\alpha_{2}}{8 \pi} W_{\mu \nu}^{i} \tilde{W}^{i \mu \nu}+C_{B} \frac{\alpha_{1}}{8 \pi} B_{\mu \nu} \tilde{B}^{\mu \nu}\right)
$$

which implies the relations

$C_{\gamma \gamma}=C_{W}+C_{B}, \quad C_{W W}=C_{W}, \quad C_{Z Z}=C_{W}+\tan ^{4} \theta_{W} C_{B}, \quad C_{Z \gamma}=C_{W}-\tan ^{2} \theta_{W} C_{B}$,

and equivalently for $\Phi=\sigma$.

Finally, let us note that qualitatively, most of the phenomenological features discussed above are fairly independent of the benchmark values $m_{\Phi} \approx 750 \mathrm{GeV}$ and $\mu_{13}^{\gamma \gamma} \approx 7 \mathrm{fb}$ associated with the diphoton excess. Given a resonance mass large enough to kinematically allow any of the decays considered above, again at a qualitative level the interplay of the dijet, $V V$ and $h h$ constraints with the diphoton signal is independent of the mass, since the widths into diboson final states scales with the same power of $m_{\Phi}$, see eqs. (1.8) and (1.9). The interplay with $t \bar{t}$ searches depends instead on $m_{\Phi}$, and this constraint becomes less important for heavier resonances. However, we will discuss both cases where fermionic decays play a role and where they do not, sections 2 and 3 respectively. We expect these two cases to be representative of the possible phenomenology of a resonance whose leading signature is in diphotons.

\section{Pseudo-Goldstone boson}

From the insights of the previous section, we are led to consider the case where $\Phi=\eta$ is a SM singlet that emerges as the GB of a spontaneously broken internal $\mathrm{U}(1)_{\eta}$ symmetry. As we show below, we need such a $\mathrm{U}(1)_{\eta}$ to be anomalous under $\mathrm{U}(1)_{Y}$ (and/or $\mathrm{SU}(2)_{L}$ ), while $\mathrm{SU}(3)_{C}$ anomalies could also be present. This type of state could find its realization 
in composite Higgs models where the pattern of global symmetry breaking $G / H$ gives rise to the Higgs and the singlet as GBs, ${ }^{2}$ and it is such that it admits a Wess-Zumino-Witten term (see e.g. the discussion in [21]). Examples can be found in models based on the coset $\mathrm{SU}(3) \times \mathrm{U}(1) / \mathrm{SU}(2) \times \mathrm{U}(1)$ [22-24], $\mathrm{SU}(4) / \mathrm{Sp}(4)$ [21, 25-29], $\mathrm{SU}(5) / \mathrm{SO}(5)$ [30-32], $\mathrm{SU}(3)^{2} / \mathrm{SU}(3)[33,34]$ like in QCD (for which the pion has an electromagnetic anomaly), and their extensions to higher-rank groups (see [35] for a review). ${ }^{3}$ In these examples, for which $\mathrm{SU}(3)_{C}$ is factorized from the coset structure, there are no colour anomalies for the GBs. These could arise however in more involved models where the colour symmetry group is embedded in a non-trivial way. Besides, we show below that a colour anomaly, in contrast to the electromagnetic one, is not strictly required to reproduce the excess.

The linear coupling of $\eta$ are given by the effective lagrangian

$$
\begin{aligned}
\mathcal{L}_{I}^{\eta}= & -\frac{\eta}{f}\left(i C_{t} \frac{\sqrt{2} m_{t}}{v} \bar{q}_{L} \tilde{H} t_{R}+\text { h.c. }+\ldots\right) \\
& -\frac{\eta}{f}\left(C_{G} \frac{\alpha_{3}}{8 \pi} G_{\mu \nu}^{a} \tilde{G}^{a \mu \nu}+C_{W} \frac{\alpha_{2}}{8 \pi} W_{\mu \nu}^{i} \tilde{W}^{i \mu \nu}+C_{B} \frac{\alpha_{1}}{8 \pi} B_{\mu \nu} \tilde{B}^{\mu \nu}\right) .
\end{aligned}
$$

which reduces to the parametrization of eq. (1.4) putting the Higgs to its VEV, keeping in mind the relations given in eq. (1.12) and $C_{g g}=C_{G}$. The top Yukawa-like coupling $C_{t}$ in eq. (2.1) (and likewise the couplings to other SM fermions) breaks the shift symmetry $\eta \rightarrow \eta+c$, and so do the anomalous terms $C_{G, W, B}$ in eq. (2.2). Therefore such terms generically contribute to the mass of the GB, see the discussion at the end of the section. Notice that another term can be added to the lagrangian of $\eta$, the standard coupling of the GB to the matter part of the broken current:

$$
\frac{1}{f} \partial_{\mu} \eta J_{\text {matter }}^{\mu}=\frac{\partial_{\mu} \eta}{f}\left[c_{q_{L}} \bar{q}_{L} \gamma^{\mu} q_{L}+c_{t_{R}} \bar{t}_{R} \gamma^{\mu} t_{R}+\ldots+c_{H}\left(i H^{\dagger}\left(D^{\mu} H\right)+\text { h.c. }\right)\right] .
$$

The effects of such terms on the phenomenology of $\eta$ can be derived by the field redefinition

$$
q_{L} \rightarrow e^{i c_{q_{L}} \eta / f} q_{L}, \quad t_{R} \rightarrow e^{i c_{t_{R}} \eta / f} t_{R}, \quad H \rightarrow e^{i c_{H} \eta / f} H, \quad \ldots
$$

which changes the action as

$$
S \rightarrow S+\int d^{4} x \frac{\eta}{f} \partial_{\mu} J^{\mu}, \quad \partial_{\mu} J^{\mu}=\partial_{\mu} J_{\text {matter }}^{\mu}+\mathcal{A}^{\eta a b} \frac{\alpha_{V}}{16 \pi} V_{\mu \nu}^{a} V_{\rho \sigma}^{b} \epsilon^{\mu \nu \rho \sigma},
$$

where $\mathcal{A}^{\eta a b}=\operatorname{Tr}\left[T^{a}\left\{T^{b}, Q^{\eta}\right\}\right]$, and $Q^{\eta}=c_{q_{L}},-c_{t_{R}}, \ldots$ are the charges associated with the transformation (2.4) expressed for left-handed chiral fermions, and $\operatorname{Tr}\left[T^{a} T^{b}\right]=\delta^{a b} / 2$ in the fundamental representation. The coupling to the current thus contributes to the Yukawas and to the anomalies in eqs. (2.1) and (2.2). ${ }^{4}$

\footnotetext{
${ }^{2}$ Generic composite Higgs models also predict the existence of extra scalar resonances, whose mass however is expected to be $m_{*}=g_{*} f$ and thus above the TeV, along with most of the strongly coupled resonances.

${ }^{3}$ Notice that some of these cosets are not endowed with a custodial symmetry, like $\mathrm{SU}(3) \times \mathrm{U}(1) / \mathrm{SU}(2) \times$ $\mathrm{U}(1)$ or $\mathrm{SU}(3)^{2} / \mathrm{SU}(3)$. Besides, in some of the models and depending on the specific realization, the singlet does not have an electromagnetic anomaly, like in [21].

${ }^{4}$ The specific contributions are $\Delta C_{t}=-c_{q_{L}}+c_{t_{R}}-c_{H}, \Delta C_{G}=-\frac{1}{2}\left(2 c_{q_{L}}-c_{t_{R}}\right), \Delta C_{W}=-\frac{3}{2} c_{q_{L}}$, and $\Delta C_{B}=-\left(\frac{1}{6} c_{q_{L}}-\frac{4}{3} c_{t_{R}}\right)$.
} 

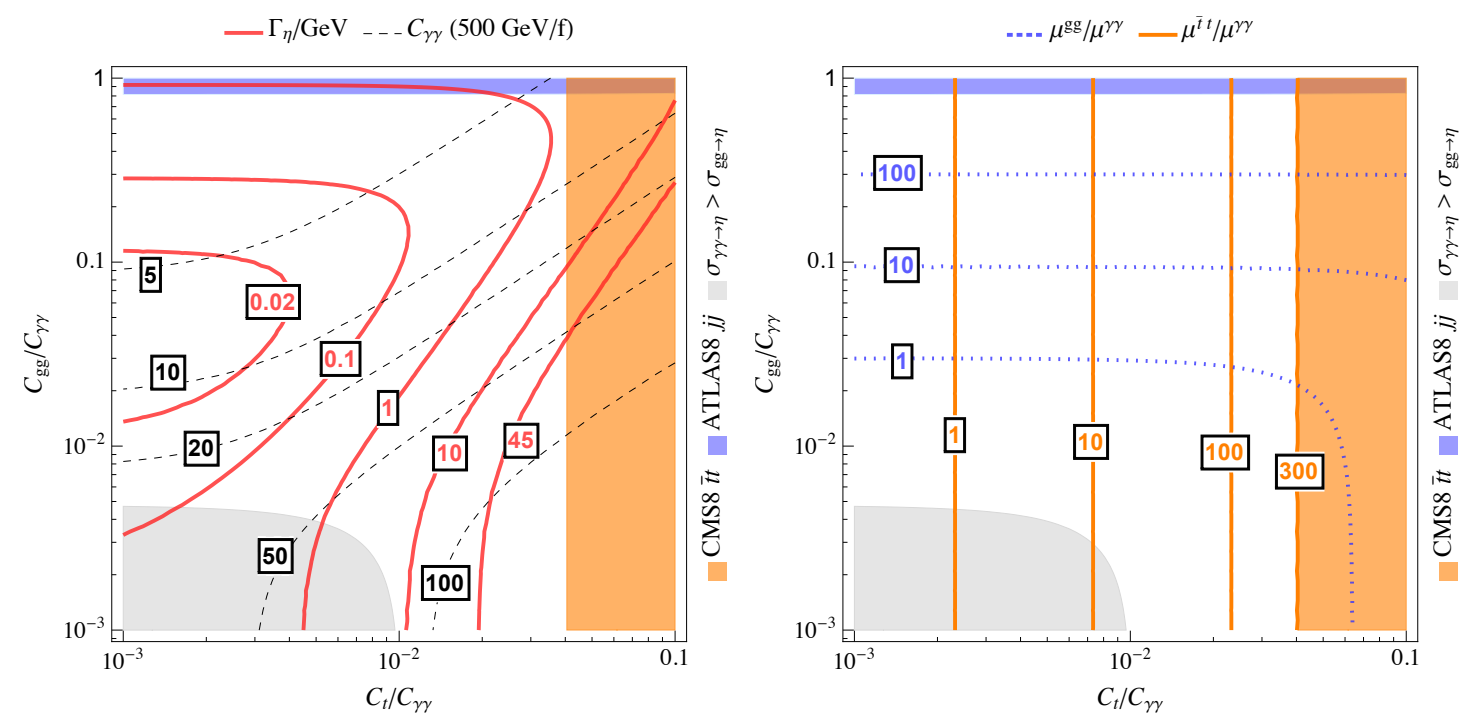

Figure 1. Pseudo-GB parameter space, where we have fixed $m_{\eta}=750 \mathrm{GeV}$ and $\mu_{13}^{\gamma \gamma}=7 \mathrm{fb}$. Shaded regions are excluded by dijet (blue) and $t \bar{t}$ (orange) searches at $8 \mathrm{TeV}$. We have also shaded in grey the region where production via photon fusion dominates over gluon fusion (bottom-left corner). Left: the solid red line gives the expected width of the minimal GB model, the dashed black line the coupling to photons normalized to $f=500 \mathrm{GeV} C_{\gamma \gamma}(500 \mathrm{GeV} / f)$. Right: ratio of signals in $t \bar{t}$ (solid orange) and $g g$ (dotted blue) with respect to the signal in $\gamma \gamma$.

Invariance under $\mathrm{CP}$ forbids the tree-level coupling to the longitudinal massive gauge bosons. Hence, the pseudo-scalar GB couples to the gauge bosons, both massless and massive, at one-loop. The decay rates to photons and gluons are given by

$$
\begin{array}{rlrl}
\Gamma_{\gamma \gamma} & =\left(\frac{\alpha_{e}}{8 \pi f}\right)^{2} \frac{m_{\eta}^{3}}{\pi}\left|C_{\gamma \gamma}^{\mathrm{eff}}\right|^{2}, & & \Gamma_{g g}=8\left(\frac{\alpha_{3}}{8 \pi f}\right)^{2} \frac{m_{\eta}^{3}}{\pi}\left|C_{g g}^{\mathrm{eff}}\right|^{2}, \\
C_{\gamma \gamma}^{\mathrm{eff}}=C_{\gamma \gamma}+\frac{1}{2} C_{t} N_{c}^{(t)} Q_{t}^{2} A_{1 / 2}\left(x_{t}\right), & C_{g g}^{\mathrm{eff}}=C_{g g}+\frac{1}{4} C_{t} A_{1 / 2}\left(x_{t}\right),
\end{array}
$$

where $N_{c}^{(t)} Q_{t}^{2}=3 \times(2 / 3)^{2}, x_{t}=4 m_{t}^{2} / m_{\eta}^{2}$, and $A_{1 / 2}(x)=2 x f(x)$ with $f(x)=$ $-\frac{1}{4}\left(\log \frac{1+\sqrt{1-x}}{1-\sqrt{1-x}}-i \pi\right)^{2}$ for $x<1$ and $f(x)=\arcsin ^{2}(1 / \sqrt{x})$ for $x>1$. Notice we have only included the loop contribution from the top, which is linked to the decay rate of $\eta$ to top pairs,

$$
\Gamma_{t \bar{t}} \simeq 3 \frac{C_{t}^{2}}{8 \pi} \frac{m_{t}^{2} m_{\eta}}{f^{2}} \approx(40 \mathrm{GeV})\left(\frac{C_{t}}{2}\right)^{2}\left(\frac{500 \mathrm{GeV}}{f}\right)^{2}\left(\frac{m_{\eta}}{750 \mathrm{GeV}}\right) .
$$

This decay channel dominates the total width of $\eta$ if the coupling to tops is sizable, and a large decay rate $\Gamma_{\eta} \approx 45 \mathrm{GeV}$ can be reproduced. This requires a large UV coefficient $C_{\gamma \gamma} \gtrsim 50$ for $f=500 \mathrm{GeV}$ in order to be compatible with $t \bar{t}$ searches eq. (1.10), and to reproduced the signal strength $\mu_{13}^{\gamma \gamma} \approx 7 \mathrm{fb}$, where it is important to recall that both the signal strength and the width scale with $1 / f^{2}$. For values of $C_{\gamma \gamma}$ close to the lower bound, a non-vanishing UV coupling to gluons $C_{g g} \approx 3$ is required. However, for larger couplings to photons, $C_{\gamma \gamma} \approx 110(f / 500 \mathrm{GeV})$, the signal strength and width can be reproduced with 
the effective coupling to gluons being generated only via the top loop, that is $C_{g g}=0$. Instead, if the requirement of a large width is relaxed, smaller values of $C_{\gamma \gamma}$ and $C_{t}$ can reproduce the excess. In fact, while $C_{\gamma \gamma} \neq 0$ is always needed, the $\eta$ coupling to tops could be completely absent, as long as $C_{g g} \neq 0$. For $f=500 \mathrm{GeV}$, values as small as $C_{\gamma \gamma} \approx 5$ and $C_{g g} \approx 0.5$ could yield the right signal strength, compatibly with dijet searches eq. (1.8). Notice that if both the couplings of $\eta$ to tops and to gluons are negligible, then photon fusion becomes the dominant production mechanism, which currently appears to be in tension with $8 \mathrm{TeV}$ bounds from $\gamma \gamma$ searches. ${ }^{5}$ These points are illustrated in figure 1 lefthand plot, where contours of $\Gamma_{\eta}$ and $C_{\gamma \gamma}(500 \mathrm{GeV} / f)$ are plotted in the plane of $C_{t} / C_{\gamma \gamma}$ and $C_{g g} / C_{\gamma \gamma}$. In the right-hand plot of figure 1 we show contours of the expected signal strength (normalized to $\mu^{\gamma \gamma}$ ) for the production and decay of $\eta$ into $t \bar{t}$ and $g g$. These channels could provide very valuable information on the GB explanation of the diphoton anomaly. ${ }^{6}$ For example, a sensitivity to top pair production cross sections below $\sim 2 \mathrm{pb}$ (assuming $\mu_{13}^{\gamma \gamma}=7 \mathrm{fb}$ ) would probe regions of parameter space still unexplored, along with the possibility that a large width is generated by decays to gauge bosons and $t \bar{t}$ alone.

In the discussion above we have omitted the couplings of the GB to $Z Z, Z \gamma$, and $W^{+} W^{-}$, all of them linked to the coupling to $\gamma \gamma$, see eq. (1.12). We have done so for simplicity of the exposition, to focus on the minimal ingredients required for the GB to reproduce the diphoton excesss. Indeed, one can explicitly check that if the GB's anomalous terms are such that $C_{B} \gg C_{W}$, the constrains from $8 \mathrm{TeV}$ searches into these other channels are easily evaded (notice the $\tan \theta_{W}$ suppression of $C_{Z Z}$ and $C_{Z \gamma}$ compared to $C_{\gamma \gamma}$ in this limit). Figure 1 has been drawn in this limit, i.e. setting $C_{W}=0$ in the relations eq. (1.12), and neglecting the top loop contribution to the $Z Z, Z \gamma, W W$ decays, since this becomes important for our discussion only in a region where $C_{t}$ is very suppressed. This however should not be taken as a solid feature of the model, on the contrary $13 \mathrm{TeV}$ searches of $g g \rightarrow \eta \rightarrow Z Z, Z \gamma, W^{+} W^{-}$should be an important target for future experimental analyses.

Finally, let us discuss the origin and the expected size of the pGB anomaly terms and mass. The size of the anomaly coefficients needed to reproduce the diphoton excess indicates that there should be a relatively large number of UV degrees of freedom charged under the SM gauge groups contributing to the anomalies. This is actually plausible in large- $N$ theories with non-trivial colour and electromagnetic charges, such as composite Higgs models with partial compositeness [39] and their holographic realizations [40-42]. In such kind of models, besides the aforementioned contributions from UV constituents, light IR d.o.f.'s like the composite top partners could contribute significantly at one-loop to the $\eta F_{\mu \nu} \tilde{F}^{\mu \nu}$ effective couplings. However, one should recall that this type of operators explicitly break the $\mathrm{U}(1)_{\eta}$ shift symmetry, and since $\eta$ is SM neutral, extra couplings breaking explicitly the symmetry, such as $C_{t} y_{t}, y_{t}=m_{t} \sqrt{2} / v$, must be involved in the generation of the loop-induced $\eta$ interactions [43]. Therefore the GB nature of $\eta$ implies that the IR contributions scale as $\Delta C_{\gamma \gamma} \sim C_{t} y_{t} f / m_{*}$, where $m_{*}$ is the mass of the composites. Most likely these contributions are too small to account for the $C_{\gamma \gamma}$ coupling required to

\footnotetext{
${ }^{5}$ This statement is, at present, subject of discussion: while the ratio $\sigma_{13 \mathrm{TeV}} / \sigma_{8 \mathrm{TeV}}$ extracted from photon parton distribution functions is close to 2 (see e.g. [37, 38]), and thus excluded, see the claim in [37] that the finite size of the proton could lift the same ratio to $\approx 4$.

${ }^{6}$ For an earlier study of similar signatures at the LHC of axion-like particles see e.g. [36].
} 
explain the diphoton signal (given $m_{*} \gtrsim f$ ), although they could gives rise to the required $C_{g g}$ coupling in some regions of the parameter space. Importantly, this is in contrast with the contributions from vector-like fermions to the couplings of a generic (pseudo-)scalar particle. In such a case loops of enough states with sufficiently large SM charges could give rise to the required $g g$ and $\gamma \gamma$ couplings, but at the expense of uncontrollable contributions to the mass of the diphoton candidate, generically pushing it to the cutoff of the effective theory [44]. In this respect, the mass of the GB is controlled by the interactions that explicitly break the global $\mathrm{U}(1)_{\eta}$ symmetry. In our minimal case we have two candidate sources: the anomalies and the top Yukawa. Other possible breaking terms can be taken naturally small, being controlled by a symmetry. The anomaly terms, even though they may come with sizeable coefficients, are totally harmless and contribute very little, if at all, to the mass of the pGB. Indeed, the anomalous coupling to photons is invariant under the shift symmetry $\eta \rightarrow \eta+c$ up to a boundary term that can be dropped given the trivial topological structure of $\mathrm{U}(1)_{\mathrm{em}}$. The anomalous coupling to gluons would seem more problematic as the action admits topologically non-trivial stationary configurations that vanish only as $1 / r$, aka instantons, for which the boundary integral gives a finite contribution. However, since the relevant scale in this case is $\Lambda_{\mathrm{QCD}}$, which is smaller than $f$ by several orders of magnitudes, the effect on the $\eta$ mass is totally negligible scaling as $\Delta m_{\eta}^{2} \sim m_{u} \Lambda_{\mathrm{QCD}}^{3} / f^{2}$. This insensitivity to sizeable anomalies for the pGB should be contrasted with the case of the dilaton discussed in section 4, see eq. (4.7). Whether the Yukawa terms in eq. (2.1) contribute or not to the mass is a model dependent assumption which is reflected on the non-linear terms in $\eta$, which we have not specified. For example, should the Yukawa of the top quark be completed by $-e^{i C_{t} \eta / f} y_{t} \bar{q}_{L} \tilde{H} t_{R}$, we could rotate away $\eta$ with a field redefinition of the type (2.4) at the expense of generating a symmetry preserving coupling to the current eq. (2.3), which has no effect on the mass, as well as the anomalous couplings to photons and gluons which has little or no effect on the mass as we have already discussed (in this case $\eta$ is essentially a heavy axion-like particle). On the other hand, should the non-linear structure for $\eta$ be such that the linear coupling of $\eta$ to the top is physical, the Yukawa would be a genuine extra source of breaking: the associated contribution to the mass, similar to the top loop contribution to the Higgs mass, can be estimated as

$$
\left(\Delta m_{\eta}^{2}\right)_{t} \sim 3 \frac{C_{t}^{2} y_{t}^{2}}{8 \pi^{2}} m_{*}^{2} \approx(775 \mathrm{GeV})^{2}\left(\frac{C_{t}}{2}\right)^{2}\left(\frac{m_{*}}{2 \mathrm{TeV}}\right)^{2} .
$$

Such a contribution disfavours $C_{t} \gg 1$. Nonetheless, it is interesting that with the same value $C_{t} \approx 2$ both the pGB mass and large width, eq. (2.8), can be naturally reproduced. This fact crucially hinges on the relatively low value $f \approx 500 \mathrm{GeV}$, given that $\Gamma_{t \bar{t}} / m_{\eta} \sim$ $\left(v^{2} / f^{2}\right)\left(m_{\eta}^{2} / f^{2}\right)$. Notice that $v^{2} / f^{2}$ is a measure of fine-tuning in the Higgs sector, thus low values of the GB decay constant are preferred (as long as they are compatible with precision measurements).

\section{Sgoldstino}

We discuss now a model where $\Phi$ has a tree-level size coupling to photons and gluons. We identify $\Phi$ with the sgoldstino, the partner of the goldstino $G$ that emerges from the 
spontaneous breaking of $\mathcal{N}=1$ SUSY. For earlier studies of sgoldstino phenomenology see e.g. [45-52] and references therein. The sgoldstino and the goldstino live in a chiral superfield $X=\Phi+\sqrt{2} \theta G+\theta^{2} F_{X}$, which is a gauge singlet that gets VEV in the auxiliary field component $\left\langle F_{X}\right\rangle=\mathcal{F} . \Phi=(\sigma+i \eta) / \sqrt{2}$ has a CP-even and a CP-odd component, $\sigma$ and $\eta$ respectively. We assume low-scale SUSY breaking, $\sqrt{\mathcal{F}} \ll M_{\mathrm{Pl}}$ (and in fact $\sqrt{\mathcal{F}}$ in the TeV range), such that the transverse gravitino components decouple and we can treat SUSY as a global symmetry in accordance to the supersymmetric equivalence theorem [53-57]. The sgoldstino is not protected by the Goldstone shift symmetry and gets a mass $\sim \mathcal{F} / M$ where $M$ is the characteristic scale of the heavy states that mediate SUSY breaking. However, the overall size of the coefficient is model dependent and it can arise either at loop- or tree-level (the difference being blurred in strongly coupled models). As for every Goldstone multiplet, the interactions originate from the coupling to the conserved (super)current. Equivalently, the couplings can be read off the soft breaking terms of SUSY, which are compensated by the would-be spurion $X$ which has been promoted to a dynamical field. For example, the gaugino masses $m_{i}$ as well as the sgoldstino couplings to gauge bosons come from

$$
-\frac{1}{2 \mathcal{F}} \int d^{2} \theta X\left(m_{1} W^{\alpha} W_{\alpha}+m_{2} W^{\alpha a_{2}} W_{\alpha}^{a_{2}}+m_{3} W^{\alpha a_{3}} W_{\alpha}^{a_{3}}\right)+\text { h.c. . }
$$

Analogously, the top $A$-term gives the stop mixing mass and a Yukawa coupling to $\Phi$ :

$$
-\frac{A_{t}}{\mathcal{F}} \int d^{2} \theta X Q_{L} H_{u} t_{R}+\text { h.c. . }
$$

With the $A$-terms proportional to the Higgs Yukawa couplings, only $A_{t}$ is potentially relevant. For concreteness, we focus hereafter on the phenomenology of the CP-even component of the sgoldstino, $\Phi=\sigma$. Picking then the $\sigma$ component of $X$, we generate possibly large couplings to the transverse gauge bosons which, in terms of the parametrization of eq. (1.3), read

$$
\begin{aligned}
\frac{c_{g g}}{f} & =\frac{2 \sqrt{2} \pi m_{3}}{\alpha_{3} \mathcal{F}}, & \frac{c_{\gamma \gamma}}{f} & =\frac{2 \sqrt{2} \pi m_{\sigma \gamma \gamma}}{\alpha_{e} \mathcal{F}}, \\
\frac{c_{W W}}{f} & =\frac{2 \sqrt{2} \pi m_{2}}{\alpha_{2} \mathcal{F}}, & \frac{c_{Z Z}}{f} & =\frac{2 \sqrt{2} \pi m_{\sigma Z Z}}{\alpha_{2} \cos ^{2} \theta_{W} \mathcal{F}},
\end{aligned}
$$

where $m_{\sigma \gamma \gamma}=m_{1} \cos ^{2} \theta_{W}+m_{2} \sin ^{2} \theta_{W}, m_{\sigma Z Z}=m_{1} \sin ^{2} \theta_{W}+m_{2} \cos ^{2} \theta_{W}$, and $m_{\sigma Z \gamma}=$ $\left(m_{2}-m_{1}\right) \sin \theta_{W} \cos \theta_{W}$. Notice that all these effective couplings scale with the ratios $m_{i} / \mathcal{F}$. The partial widths into gauge bosons, at leading order in $m_{V} / m_{\sigma}$, then read

$$
\begin{aligned}
\Gamma_{g g} & =\left(\frac{m_{3}}{2 \mathcal{F}}\right)^{2} \frac{m_{\sigma}^{3}}{\pi}, & \Gamma_{\gamma \gamma} & =\frac{1}{2}\left(\frac{m_{\sigma \gamma \gamma}}{4 \mathcal{F}}\right)^{2} \frac{m_{\sigma}^{3}}{\pi}, \\
\Gamma_{Z Z} & \simeq \frac{1}{2}\left(\frac{m_{\sigma Z Z}}{4 \mathcal{F}}\right)^{2} \frac{m_{\sigma}^{3}}{\pi}, & \Gamma_{W W} \simeq\left(\frac{m_{2}}{4 \mathcal{F}}\right)^{2} \frac{m_{\sigma}^{3}}{\pi}, & \Gamma_{Z \gamma} \simeq\left(\frac{m_{\sigma Z \gamma}}{4 \mathcal{F}}\right)^{2} \frac{m_{\sigma}^{3}}{\pi} .
\end{aligned}
$$

Other subleading contributions come from the coupling to longitudinal vector bosons, $\sigma V_{\mu} V^{\mu}$, see for instance [45, 49]. A contribution to such interactions comes from a mixing 
of $\sigma$ with the Higgs boson, controlled e.g. by the $\mu$-term. However for $\mu / m_{1,2} \lesssim 1 / 2$ the corrections to the partial widths above are below the experimental sensitivity. As a matter of fact, to leading order in the gauge boson masses, the expressions above are still valid with the replacement $m_{\sigma Z Z}^{2} \rightarrow m_{\sigma Z Z}^{2}+\mu^{2} / 2$ for $\Gamma_{Z Z}$ (and $m_{2}^{2} \rightarrow m_{2}^{2}+\mu^{2} / 2$ for $\Gamma_{W W}$ as well). The $\mu$-term in these replacements is actually the mass entry of the neutral Higgsinos, $\mu \tilde{H}_{1}^{0} \tilde{H}_{2}^{0}$, which is part of the full neutralino mass matrix that can be found in [47]. A sizeable mixing with the Higgs may be problematic to explain the supposed signal, given the bounds at $8 \mathrm{TeV}$, in particular those from $Z Z$ and $W^{+} W^{-}$resonance searches. We work here in the limit where this mixing is negligible, an assumption justified also by measurements of the Higgs signal strengths, in particular in the diphoton channel. The widths translate into the following ratios of decay rates

$$
r_{g g}^{\gamma}=\frac{1}{8} \frac{m_{\sigma \gamma \gamma}^{2}}{m_{3}^{2}}, \quad r_{Z Z}^{\gamma} \simeq \frac{m_{\sigma \gamma \gamma}^{2}}{m_{\sigma Z Z}^{2}}, \quad r_{Z \gamma}^{\gamma} \simeq \frac{1}{2} \frac{m_{\sigma \gamma \gamma}^{2}}{m_{\sigma Z \gamma}^{2}}, \quad r_{W W}^{\gamma} \simeq \frac{1}{2} \frac{m_{\sigma \gamma \gamma}^{2}}{m_{2}^{2}},
$$

which are a priori well suited to fit the diphoton excess consistently with $8 \mathrm{TeV}$ searches. From these ratios the constraints on $r_{V V}^{\gamma}$ and $r_{g g}^{\gamma}$ in table 2 can be easily satisfied in a region of parameter space where $m_{3} / m_{1}$ and $m_{2} / m_{1}$ are not too large, as shown in figure 2 . It is important to notice that the sgoldstino mass term implies an irreducible invisible width because of the decay channel into goldstinos (aka the longitudinal polarizations of the gravitino after coupling SUSY to gravity [53-55])

$$
\Gamma_{\mathrm{inv}} \simeq \frac{m_{\sigma}^{5}}{32 \pi \mathcal{F}^{2}}, \quad r_{\mathrm{inv}}^{\gamma} \simeq \frac{m_{\sigma \gamma \gamma}^{2}}{m_{\sigma}^{2}},
$$

which originates from the soft SUSY breaking term $-m_{\sigma}^{2} /\left(4 \mathcal{F}^{2}\right) \int d^{4} \theta\left(X^{\dagger} X\right)^{2}$. The constraint on $r_{\text {inv }}^{\gamma}$ from invisible decays of $\sigma$ (which can be derived from the monojet searches of [58]) is very mild, and automatically satisfied given the existing lower bounds on gaugino masses. As a matter of fact, this width is typically small and we have neglected it in obtaining figure 2 .

The production cross section of the sgoldstino scales proportionally to $\left(m_{3} / \mathcal{F}\right)^{2}$, and the signal strength can be written as

$$
\mu_{13, \sigma}^{\gamma \gamma} \simeq 7 \mathrm{fb}\left(\frac{14 \mathrm{TeV}}{\sqrt{\mathcal{F}}}\right)^{4}\left(\frac{m_{3}}{3 \mathrm{TeV}}\right)^{2} \frac{1}{\left(m_{3} / m_{2}\right)^{2}+1 / 2+0.07\left(\mathrm{TeV} / m_{2}\right)^{2}},
$$

where for simplicity in this formula we have assumed $m_{1}=m_{2}$. In the left-hand plot of figure 2 we show isolines (dashed black) for the values of $\mathcal{F} /\left(\mathrm{TeV} \cdot m_{3}\right)$ that correspond to signal strength that best fits the excess. Dijet searches at the LHC Run-1 require $\mathcal{F} /\left(\mathrm{TeV} \cdot m_{3}\right) \gtrsim 6$, which translates into a lower bound on the gluino mass. Such a bound may or may not be relevant depending on the specific SUSY breaking model. Notice that $Z Z$ searches at $8 \mathrm{TeV}$ exclude the region where photon fusion (plus possibly $Z Z$ and $Z \gamma$ production) dominates over gluon fusion. The sgoldstino total width can be written as

$$
\Gamma_{\sigma} \simeq 8 \mathrm{MeV}\left(\frac{14 \mathrm{TeV}}{\sqrt{\mathcal{F}}}\right)^{4}\left(\frac{m_{3}}{3 \mathrm{TeV}}\right)^{2}\left[1+\frac{1}{2}\left(m_{2} / m_{3}\right)^{2}+0.07\left(\mathrm{TeV} / m_{3}\right)^{2}\right],
$$



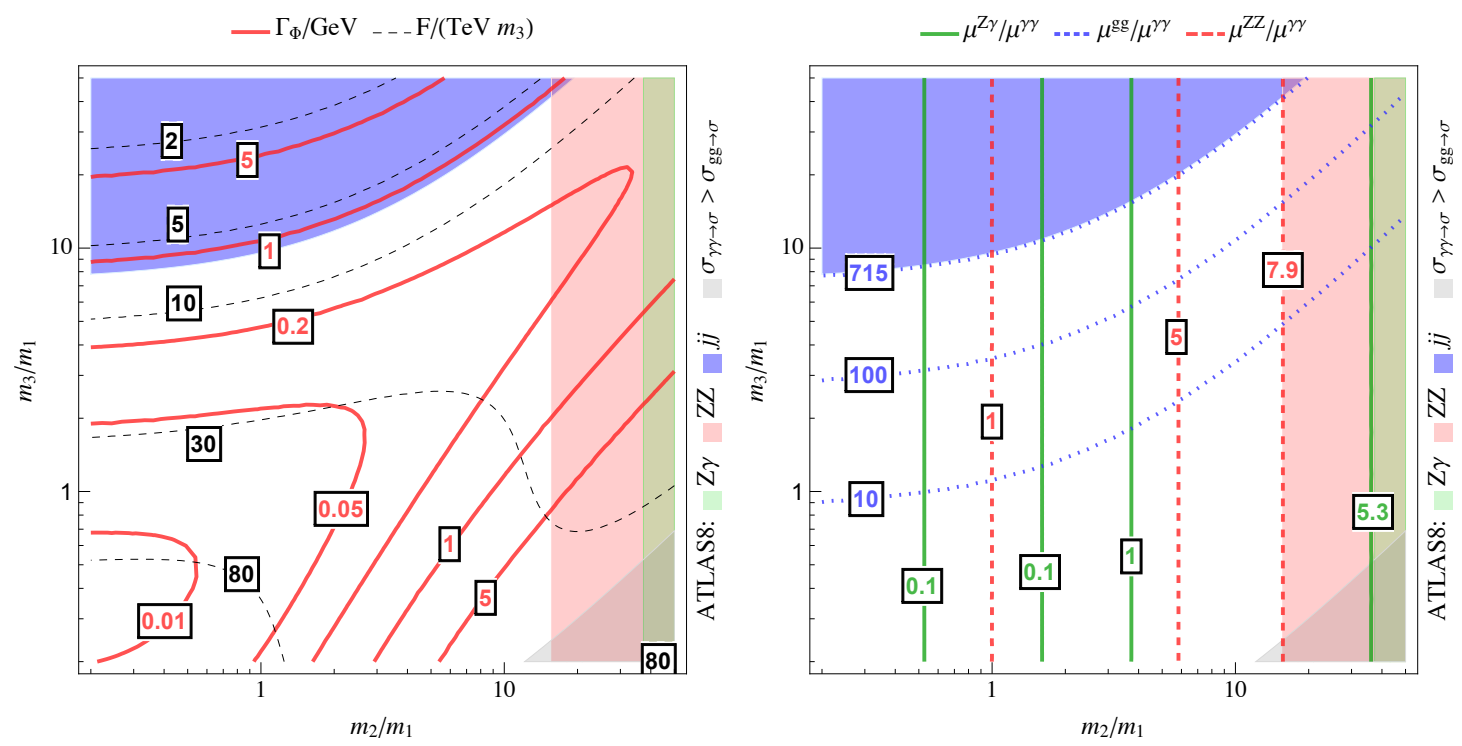

Figure 2. Sgoldstino parameter space, where we have fixed $m_{\sigma}=750 \mathrm{GeV}$ and $\mu_{13}^{\gamma \gamma}=7 \mathrm{fb}$. Shaded regions are excluded by dijet (blue), $Z Z$ (red) and $Z_{\gamma}$ (green) searches at $8 \mathrm{TeV}$. We have also shaded in grey the region where production via photon fusion dominates over gluon fusion (bottomright corner). Left: the solid red line gives the expected width of the minimal sgoldstino model, the dashed black line the scale $\mathcal{F} /\left(\mathrm{TeV} \cdot m_{3}\right)$. Right: ratio of signals in $Z Z$ (dashed red), $Z \gamma$ (solid green) and $g g$ (dotted blue) with respect to the signal in $\gamma \gamma$.

so that the generic expectation is that the minimal sgolstino appears as a narrow resonance. Contours of fixed widths, again with the assumption of negligible invisible width, are shown as thick red lines in the left-hand plot of figure 2. These values are too small compared with the preferred width determination by ATLAS. However, given the little amount of data currently available, we do not consider this as a major drawback of the sgoldstino model, and we postpone a more careful analysis of the total width to when more data is available (including an error for the measurement of the width). Likewise, we leave for future work the exploration of the parameter regions of the scalar potential and/or non-minimal models with an extended Higgs sector beyond the MSSM, where it could be possible to generate a larger width in invisible or other channels, resulting in a broader resonance. Finally, in the right-hand plot of figure 2, we show the signal expected in the most promising channels to confirm the hypothesis of the sgoldstino, if this is indeed responsible for the $750 \mathrm{GeV}$ diphoton excess. This plot can provide a useful guidance to experimental collaborations, providing sensitivity targets for dijet, $Z Z$ and $Z \gamma$ resonance searches. For example, a sensitivity to cross sections in $Z \gamma$ below $\sim 20 \mathrm{fb}$ (assuming $\mu_{13}^{\gamma \gamma}=7 \mathrm{fb}$ ) would probe regions of parameter space that are still unexplored, and constrain the gaugino spectrum.

While we focussed on the CP-scalar $\sigma$ in $X$, its CP-odd component $\eta$ has very similar coupling structures, dictated as well by the soft SUSY breaking terms. The phenomenology of such pseudo-scalar is fully analogous to the analysis we presented above for $\sigma$. 


\section{Dilaton}

In the presence of large diphoton rates, the dilaton, i.e. the GB of spontaneously broken conformal symmetry, is perhaps one of the first scalar resonances that comes to mind in the context of strongly coupled models. ${ }^{7}$ Indeed, the dilaton has potentially sizeable couplings to massless gauge bosons, controlled by the $\beta$-function contributions from the states of the conformal field theory (CFT), see e.g. [59-67]. We consider a strongly coupled CFT with a global $\mathrm{SU}(3)_{C} \times \mathrm{SU}(2)_{L} \times \mathrm{U}(1)_{Y}$ symmetry, whose associated conserved currents weakly couple to gluons and electroweak gauge bosons. The current central charges $\kappa_{J}$ control the $\beta$-functions contributions

$$
\left\langle J_{\mu}^{a}(p) J_{\nu}^{b}(-p)\right\rangle_{\mathrm{CFT}}=\delta^{a b}\left(\eta_{\mu \nu} p^{2}-p_{\mu} p_{\nu}\right) \frac{\kappa_{J}}{16 \pi^{2}} \log p^{2}+\ldots, \quad \beta_{J}^{\mathrm{CFT}}=\kappa_{J} \frac{g_{J}^{3}}{16 \pi^{2}},
$$

where $g_{J}$ is the relevant gauge coupling between the gauge field and its current $J_{\mu}$. The central charge roughly counts how many degrees of freedom in the CFT are charged under the symmetry. Spontaneous breaking of conformality generates a light dilaton $\sigma$ with its decay constant $f$. The compensator $\sigma / f$ must match in the IR the trace anomaly contribution from the CFT triggered by weak gauging in the UV

$$
\frac{\sigma}{f} T_{\mu}^{\mu \mathrm{CFT}}=\frac{\sigma}{f}\left(\frac{\alpha_{3}}{8 \pi} \kappa_{3} G_{\mu \nu}^{2}+\kappa_{e} \frac{\alpha_{e}}{8 \pi} F_{\mu \nu}^{2}-y_{t}\left(1+\epsilon_{t}\right) \bar{q}_{L} \tilde{H} t_{R}+\text { h.c. }+2\left(1+\epsilon_{H}\right)\left|D_{\mu} H\right|^{2}+\ldots\right) .
$$

We have also included in the trace the contributions coming from the terms responsible for generating the masses of matter fields and electroweak gauge boson, where the electroweak symmetry is spontaneously broken by a Higgs operator $H$, which should be part of the CFT in order not to introduce a hierarchy problem. Within partial compositeness [39], elementary fermions $\psi_{L, R}$ probe the CFT by mixing in the UV with some fermionic operators $\Psi_{R, L}^{C F T}$ of scaling dimension $\Delta_{L, R}=5 / 2+\gamma_{L, R}$, with the strength of the interaction set by the proto-Yukawas $y_{L, R}$. The resulting IR Yukawa coupling for the massless SM fermion emerging from the mixing scales as $y_{f} \sim y_{L} y_{R}$. Dilaton insertions $e^{\Delta_{f} \sigma / f}$ must then compensate in the IR a dimension $\Delta_{f}=1+\epsilon_{f}$, where $\epsilon_{f}=\gamma_{L}+\gamma_{R}$. We will assume in the following that for the case of the top quark $\epsilon_{t}=0$, although this is not an essential (nor very relevant) feature of the model. ${ }^{8}$ Analogously, one must insert $e^{2 \sigma / f}$ to compensate for the gauge bosons mass terms, of conformal weight equal to two, with possible departures parametrized by $\epsilon_{H}$. We have further assumed that electroweak symmetry breaking proceeds while respecting custodial symmetry, which enforces $c_{W}=c_{Z}=c_{h}$ in eq. (1.3). Moreover, in composite Higgs models where $H$ arises as a pseudo-Goldstone boson of the strong dynamics one obtains $\epsilon_{H} \simeq 0$, since such a Higgs is fully composite and a possible Higgs-dilaton kinetic mixing is suppressed by the Higgs' shift symmetry [69, 70] (we come back to this point below). All in all, for the minimal and natural dilaton model we get

$$
c_{g g}=\kappa_{3}, \quad c_{\gamma \gamma}=\kappa_{e}, \quad c_{W}=c_{Z}=c_{h} \simeq c_{t} \simeq 1,
$$

\footnotetext{
${ }^{7}$ We should remark that explicit constructions of spontaneous breaking of conformality, without SUSY, require strong dynamical assumptions [64, 65, 68], see [71-75] for explicit realizations.

${ }^{8}$ The fermionic anomalous dimensions $\gamma_{L, R}$ depend on the particular model of flavour, and special cases such as $\epsilon_{t} \simeq-1$ could also be realized.
} 
for the parameters in the effective lagrangian in eq. (1.3). The effective couplings to photons and gluons are then given by

$$
c_{\gamma \gamma}^{\mathrm{eff}}=c_{\gamma \gamma}+c_{t} N_{c}^{(t)} Q_{t}^{2} F_{1 / 2}\left(x_{t}\right)-c_{W} F_{1}\left(x_{W}\right), \quad c_{g g}^{\mathrm{eff}}=c_{g g}+c_{t} \frac{1}{2} F_{1 / 2}\left(x_{t}\right)
$$

where $N_{c}^{(t)} Q_{t}^{2}=3 \times(2 / 3)^{2}, x_{i}=4 m_{i}^{2} / m_{\sigma}^{2}$, and $F_{1 / 2}(x)=2 x[1+(1-x) g(x)], F_{1}(x)=$ $2+3 x+3 x(2-x) g(x)$ with $g(x)=\left[\sin ^{-1}(1 / \sqrt{x})\right]^{2}$. The coefficients in eq. (4.4) set the decay rates for $\sigma \rightarrow \gamma \gamma$ and $\sigma \rightarrow g g$ as in the pGB case, eq. (2.6), with $m_{\eta} \rightarrow m_{\sigma}$ and $C_{g g, \gamma \gamma}^{\mathrm{eff}} \rightarrow c_{g g, \gamma \gamma}^{\mathrm{eff}} / 2$. The unknown UV contributions to $c_{g g, \gamma \gamma}^{\mathrm{eff}}$, parametrized by $\kappa_{3}$ and $\kappa_{e}$, allow us to treat these coefficients as free parameters in this model. Actually, the large rates in diphotons needed to reproduce the supposed excess, consistently with $8 \mathrm{TeV}$ searches, call for values of $\kappa_{e, 3}$ that dominate the expressions for $c_{\gamma \gamma, g g}^{\mathrm{eff}}$. Hence in the following we will focus on the implications of the diphoton signal for the $\beta$-function coefficients defined in eq. (4.1).

A significant challenge for the dilaton scenario of eq. (4.3) is posed by the predicted large rate in $Z Z, W^{+} W^{-}$and $h h$,

$$
\Gamma_{Z Z} \simeq \Gamma_{W W} / 2 \simeq \Gamma_{h h} \simeq \frac{m_{\sigma}^{3}}{32 \pi f^{2}},
$$

given that the corresponding couplings are generated at tree-level, while the coupling to photons is a priori of loop-size. This implies, for the most sensitive channel at the LHC Run-1,

$$
r_{Z Z}^{\gamma} \approx 0.05\left(\frac{\kappa_{e}}{240}\right)^{2}
$$

Therefore, in order to satisfy the bounds from $Z Z$ final state searches in table 2 , the contribution from the UV d.o.f.'s of the CFT to $\kappa_{e}$ needs to roughly compensate for the smallness of $\alpha_{e}=e^{2} /(4 \pi)$. Considering interference effects, which could be non-negligible given the width of $\Phi$ that is suggested by the recent Run-2 diphoton data, the limit from $Z Z$ searches could not apply or be considerably loosen, hence opening the possibility to fit the diphoton excess with smaller values of $\kappa_{e}$. However, even in that case the combined limits from $Z Z+W^{+} W^{-}$searches, which cover the case of new physics resonances with a large width, would apply and the lower bound on $\kappa_{e}$ would be reduced only by a factor less than half, leaving unchanged the conclusion that a very large electromagnetic trace anomaly is needed to attain the needed signal rates without clashing with Run-1 searches.

Such a large electromagnetic $\beta$-function is the signal of a substantial breaking of the conformal symmetry. This feeds back into the dilaton mass, which is expected to scale as $[64,71]$

$$
\frac{m_{\sigma}^{2}}{\Lambda^{2}} \sim \alpha_{e} \kappa_{e}
$$

where $\Lambda$ is the scale associated with the heavy resonances of the strongly coupled CFT. For large $\kappa_{e} \gtrsim 1 / \alpha_{e}$ as those needed to reproduce the diphoton excess, the dilaton is then expected to be as heavy as a generic composite resonance. The calculability and selection rules associated with scale invariance, whose corrections scale precisely as $\left(m_{\sigma} / \Lambda\right)^{2}$, 
are generically lost in this case. The dilaton is then expected to behave as an ordinary composite spin-0 resonance of the strong sector, perhaps accidentally light, and which nevertheless could still be well suited to explain the diphoton excess.

One tentative solution for this problem would be to depart from the tree-level couplings of the dilaton to the massive vector bosons given in eq. (4.3). Given a modification $c_{V} \rightarrow$ $c_{V}\left(1+\delta_{V}\right)$, with $\delta_{V}<0, V=\{W, Z\}$, the ratio of $\gamma \gamma$ to $Z Z$ decay rates increases by a factor $r_{Z Z}^{\gamma} \rightarrow r_{Z Z}^{\gamma} /\left(1+\delta_{V}\right)^{2}$. The absence of a signal at the LHC Run-1 in $Z Z$ resonance searches requires quite large deviations $\delta_{V} \gtrsim 1 / 3$. Retaining the assumption that the Higgs is a pGB of the strong dynamics, such a large correction indicates that the expansion parameter of explicit conformal symmetry breaking $m_{\sigma}^{2} / \Lambda^{2} \sim \delta_{V}$ should be large. Alternatively, one could consider a composite Higgs which is not a pGB. In such a case, a kinetic dilaton-Higgs mixing of the type

$$
c H^{\dagger} D^{\mu} H \partial_{\mu} \sigma / f+h . c .
$$

results in $O(1)$ corrections to the dilaton coupling to vectors, $\delta_{V}=\delta c_{V}=-c{ }^{9}$ The constraints from Higgs couplings measurements associated to such a mixing could be evaded, since the correction to an on-shell Higgs coupling $g$ scales as $\delta g / g_{S M} \sim\left(g_{\sigma} / g_{S M}\right) \times c \times$ $(v / f)\left(m_{h} / m_{\sigma}\right)^{2}$, where $g_{\sigma}$ is the coupling of the dilaton to the same final states. For example, in the SM the Higgs coupling to photons is $g_{S M}=c_{\gamma \gamma}^{h} \approx-6.5$ at one-loop in the normalization of eq. (1.3), and due to the mixing with the dilaton it would get a relative correction $\delta g / g_{S M} \sim(v / f) \kappa_{e} /(36 \times 6.5)$, possibly below the current experimental sensitivity for the values of $\kappa_{e}$ needed to reproduce the diphoton excess. However, we should notice that the term in (4.8) breaks the Higgs's shift symmetry and therefore contributes to the Higgs potential at loop-level. For a composite Higgs without Goldstone protection, for which $c$ is sizable, one can estimate

$$
\Delta m_{H}^{2} \sim \frac{c}{16 \pi^{2}} \frac{\Lambda^{4}}{f^{2}}
$$

which contributes significantly to the tuning of the electroweak scale, $\Delta m_{H}^{2} / m_{h}^{2} \sim$ $c 600 f^{2} / v^{2}$ for $\Lambda \simeq 4 \pi f$.

\section{Conclusions}

The power of LHC Run-2 for producing new states beyond the SM offers an excellent opportunity to explore the $\mathrm{TeV}$ scale. In this work we have investigated resonance production in the diphoton channel, motivated by the recent diphoton anomaly reported by

\footnotetext{
${ }^{9}$ The dilaton-Higgs mixing term, alone, breaks also special conformal transformations $\delta x^{\mu}=2(b \cdot x) x^{\mu}-$ $b^{\mu} x^{2}+o\left(b^{2}\right)$, and it is thus forbidden in theories that are fully conformal as opposed to just scale invariant. Nevertheless, since the combination $\square \sigma / f+\left(\partial_{\mu} \sigma / f\right)^{2}$ transforms covariantly with conformal weight 2, even with respect to special conformal transformations acting as $\sigma(x) \rightarrow \sigma\left(x\left(x^{\prime}\right)\right)-f \log J^{1 / 4}\left(x\left(x^{\prime}\right)\right)$ with $J(x)=1+8 b \cdot x+o\left(b^{2}\right)$, the operator $-c|H|^{2}\left[\square \sigma / f+\left(\partial_{\mu} \sigma\right)^{2} / f^{2}\right]$ contains the dilaton-Higgs mixing term up to a total derivative and an extra interaction vertex that rescales the dilaton kinetic term by $\left(1+c v^{2} / 2 f^{2}\right)$. From this conformal covariant expression for the kinetic mixing one can readily get $\delta c_{V}=-c$ by comparing it with the conformal covariant kinetic term $e^{2 \sigma / f}\left|D_{\mu}\left(H e^{-\sigma / f}\right)\right|^{2}$ responsible for the Higgs and massive gauge bosons coupling in eq. (4.2), after the field redefinition $H \rightarrow H e^{\sigma / f}$.
} 
the ATLAS and CMS collaborations. We focussed on Goldstone bosons, well-motivated candidates given their intrinsic connection with supersymmetry and composite Higgs models. Moreover, their low-energy predictions are mostly dictated by the non-linearly realized symmetries, and their soft or anomalous breakings. Their mass is under theoretical control and naturally below the cutoff.

We studied three paradigmatic scenarios: the Goldstone boson of a spontaneously broken internal symmetry, focusing on a $\mathrm{U}(1)$, which could be part of a larger symmetry breaking pattern like in composite Higgs models; the scalar partner of the goldstino in $\mathcal{N}=1$ supersymmetry, known as the sgoldstino; and the dilaton from a conformal field theory broken spontaneously.

A CP-odd GB is well suited to fit the excess, given the suppressed coupling to longitudinal vector bosons and Higgs bosons due to $\mathrm{CP}$ invariance, and relatively large anomaly coefficients of $O(10)$. Interestingly, the production cross section and the decay rate to photons are mainly controlled by UV anomalies, which could thus potentially shed light on the UV completion of composite Higgs models. In particular, a sizable electromagnetic anomaly is unavoidable, whereas the required effective coupling to gluons could be due to a large coupling to top quarks, yielding also a large width of the CP-odd diphoton candidate.

The sgoldstino presents couplings to $\gamma \gamma, g g$, and $W W, Z Z$ and $Z \gamma$ which are all of the same order and sizable, thus being able to accommodate the excess with mild constraints on the spectrum of the gauginos. This model, in its minimal incarnation, prefers a small width, below $\sim 1 \mathrm{GeV}$. Importantly, the observation of the sgoldstino at the LHC could provide information on the mechanism of supersymmetry breaking.

The minimal and natural dilaton model is instead disfavoured by present data as it couples too strongly to the longitudinal vector bosons and to the Higgs boson, whereas the coupling to photons requires a huge trace anomaly, which in turn compromises the consistency of the model. The dilaton as a diphoton candidate could be rescued by reducing the couplings to the Higgs field, at the cost of increasing the amount of fine-tuning in the Higgs potential.

The confirmation or dismissal of any of these scenarios is a matter of future exploration and more data from the LHC. For this reason, we have provided in figures 1 and 2 the expected signals in channels other than $\gamma \gamma$, respectively for the pseudo-Goldstone boson $(j j$ and $t t)$ and the sgoldstino $(j j, Z Z$ and $Z \gamma)$.

Note added: the talks by CMS and ATLAS on the diphoton anomaly took place on Tuesday Dec. 15th [1]. Already by the end of that week a large amount of theoretical papers [37, 76-112] appeared on arXiv.org as response to this input from the experiments. Such a tremendous research activity reflects the excitement of the community from the hints of new physics that we may have finally glimpsed at the LHC.

\section{Acknowledgments}

We thank Dario Buttazzo and Diego Redigolo for useful discussions. B.B. thanks Stephan Lavignac and Anibal Medina for interesting discussions. R.F. thanks Roberto Contino 
for discussions. B.B. is supported in part by the MIUR-FIRB grant RBFR12H1MW. F.S. is supported by the European Research Council (ERC) under the EU Seventh Framework Programme (FP7/2007-2013)/ERC Starting Grant (agreement n. 278234 — 'NEWDARK' project).

Open Access. This article is distributed under the terms of the Creative Commons Attribution License (CC-BY 4.0), which permits any use, distribution and reproduction in any medium, provided the original author(s) and source are credited.

\section{References}

[1] J. Olsen and M. Kado, ATLAS and CMS physics results from Run 2, talks given at CERN, December 15 (2015), https://indico.cern.ch/event/442432/.

[2] ATLAS collaboration, Search for resonances decaying to photon pairs in $3.2 \mathrm{fb}^{-1}$ of pp collisions at $\sqrt{s}=13 \mathrm{TeV}$ with the ATLAS detector, ATLAS-CONF-2015-081 (2015).

[3] CMS collaboration, Search for new physics in high mass diphoton events in proton-proton collisions at $\sqrt{s}=13 \mathrm{TeV}$, CMS-PAS-EXO-15-004 (2015).

[4] A.D. Martin, W.J. Stirling, R.S. Thorne and G. Watt, Parton distributions for the LHC, Eur. Phys. J. C 63 (2009) 189 [arXiv:0901.0002] [inSPIRE].

[5] ATLAS collaboration, Search for high-mass diphoton resonances in pp collisions at $\sqrt{s}=8$ TeV with the ATLAS detector, Phys. Rev. D 92 (2015) 032004 [arXiv:1504.05511] [INSPIRE].

[6] CMS collaboration, Search for diphoton resonances in the mass range from 150 to $850 \mathrm{GeV}$ in pp collisions at $\sqrt{s}=8 \mathrm{TeV}$, Phys. Lett. B 750 (2015) 494 [arXiv:1506.02301] [INSPIRE].

[7] ATLAS collaboration, Search for a high-mass Higgs boson decaying to a $W$ boson pair in pp collisions at $\sqrt{s}=8 \mathrm{TeV}$ with the ATLAS detector, JHEP 01 (2016) 032 [arXiv: 1509.00389] [INSPIRE].

[8] CMS collaboration, Search for a Higgs boson in the mass range from 145 to $1000 \mathrm{GeV}$ decaying to a pair of $W$ or $Z$ bosons, JHEP 10 (2015) 144 [arXiv:1504.00936] [INSPIRE].

[9] ATLAS collaboration, Search for an additional, heavy Higgs boson in the $H \rightarrow Z Z$ decay channel at $\sqrt{s}=8 \mathrm{TeV}$ in pp collision data with the ATLAS detector, Eur. Phys. J. C 76 (2016) 45 [arXiv: 1507.05930] [INSPIRE].

[10] ATLAS collaboration, Search for new resonances in $W \gamma$ and $Z \gamma$ final states in $p p$ collisions at $\sqrt{s}=8 \mathrm{TeV}$ with the ATLAS detector, Phys. Lett. B 738 (2014) 428 [arXiv: 1407.8150] [INSPIRE].

[11] ATLAS collaboration, Searches for Higgs boson pair production in the $h h \rightarrow b b \tau \tau, \gamma \gamma W W^{*}, \gamma \gamma b b, b b b b$ channels with the ATLAS detector, Phys. Rev. D 92 (2015) 092004 [arXiv: 1509. 04670] [INSPIRE].

[12] CMS collaboration, Search for resonant pair production of Higgs bosons decaying to two bottom quark-antiquark pairs in proton-proton collisions at $8 \mathrm{TeV}$, Phys. Lett. B $\mathbf{7 4 9}$ (2015) 560 [arXiv: 1503.04114] [inSPIRE].

[13] ATLAS collaboration, A search for $t \bar{t}$ resonances using lepton-plus-jets events in proton-proton collisions at $\sqrt{s}=8 \mathrm{TeV}$ with the ATLAS detector, JHEP 08 (2015) 148 [arXiv: 1505.07018] [INSPIRE]. 


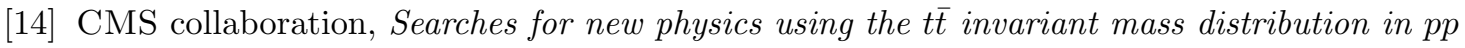
collisions at $\sqrt{s}=8$ TeV, Phys. Rev. Lett. 111 (2013) 211804 [arXiv:1309.2030] [INSPIRE].

[15] ATLAS collaboration, A search for high-mass resonances decaying to $\tau^{+} \tau^{-}$in pp collisions at $\sqrt{s}=8$ TeV with the ATLAS detector, JHEP 07 (2015) 157 [arXiv:1502.07177] [INSPIRE].

[16] CMS collaboration, Search for additional neutral Higgs bosons decaying to a pair of tau leptons in pp collisions at $\sqrt{s}=7$ and 8 TeV, CMS-PAS-HIG-14-029 (2014).

[17] ATLAS collaboration, Search for high-mass dilepton resonances in pp collisions at $\sqrt{s}=8$ TeV with the ATLAS detector, Phys. Rev. D 90 (2014) 052005 [arXiv:1405.4123] [INSPIRE].

[18] CMS collaboration, Search for physics beyond the standard model in dilepton mass spectra in proton-proton collisions at $\sqrt{s}=8 \mathrm{TeV}$, JHEP 04 (2015) 025 [arXiv:1412.6302] [INSPIRE].

[19] ATLAS collaboration, Search for new phenomena in the dijet mass distribution using $p p$ collision data at $\sqrt{s}=8$ TeV with the ATLAS detector, Phys. Rev. D 91 (2015) 052007 [arXiv: 1407.1376] [INSPIRE].

[20] CMS Collaboration, Search for resonances decaying to dijet final states at $\sqrt{s}=8$ TeV with scouting data, CMS-PAS-EXO-14-005 (2014).

[21] B. Gripaios, A. Pomarol, F. Riva and J. Serra, Beyond the minimal composite Higgs model, JHEP 04 (2009) 070 [arXiv:0902.1483] [InSPIRE].

[22] R. Contino, Y. Nomura and A. Pomarol, Higgs as a holographic pseudoGoldstone boson, Nucl. Phys. B 671 (2003) 148 [hep-ph/0306259] [INSPIRE].

[23] M. Schmaltz, The simplest little Higgs, JHEP 08 (2004) 056 [hep-ph/0407143] [INSPIRE].

[24] C.T. Hill and R.J. Hill, Topological physics of little Higgs bosons, Phys. Rev. D 75 (2007) 115009 [hep-ph/0701044] [INSPIRE].

[25] E. Katz, A.E. Nelson and D.G.E. Walker, The intermediate Higgs, JHEP 08 (2005) 074 [hep-ph/0504252] [INSPIRE].

[26] J. Galloway, J.A. Evans, M.A. Luty and R.A. Tacchi, Minimal conformal technicolor and precision electroweak tests, JHEP 10 (2010) 086 [arXiv: 1001.1361] [INSPIRE].

[27] J. Barnard, T. Gherghetta and T.S. Ray, UV descriptions of composite Higgs models without elementary scalars, JHEP 02 (2014) 002 [arXiv:1311.6562] [INSPIRE].

[28] J. Serra, Beyond the minimal top partner decay, JHEP 09 (2015) 176 [arXiv:1506.05110] [INSPIRE].

[29] H. Cai, T. Flacke and M. Lespinasse, A composite scalar hint from di-boson resonances?, arXiv: 1512.04508 [INSPIRE].

[30] N. Arkani-Hamed, A.G. Cohen, E. Katz and A.E. Nelson, The littlest Higgs, JHEP 07 (2002) 034 [hep-ph/0206021] [INSPIRE].

[31] L. Vecchi, The natural composite Higgs, arXiv:1304.4579 [INSPIRE].

[32] G. Ferretti, UV completions of partial compositeness: the case for a $\mathrm{SU}(4)$ gauge group, JHEP 06 (2014) 142 [arXiv:1404.7137] [INSPIRE].

[33] N. Arkani-Hamed et al., The minimal moose for a little Higgs, JHEP 08 (2002) 021 [hep-ph/0206020] [INSPIRE]. 
[34] L. Vecchi, A "dangerous irrelevant" UV-completion of the composite Higgs, arXiv: 1506.00623 [INSPIRE].

[35] B. Bellazzini, C. Csáki and J. Serra, Composite Higgses, Eur. Phys. J. C 74 (2014) 2766 [arXiv: 1401.2457] [INSPIRE].

[36] J. Jaeckel, M. Jankowiak and M. Spannowsky, LHC probes the hidden sector, Phys. Dark Univ. 2 (2013) 111 [arXiv: 1212.3620] [INSPIRE].

[37] S. Fichet, G. von Gersdorff and C. Royon, Scattering light by light at $750 \mathrm{GeV}$ at the LHC, arXiv: 1512.05751 [INSPIRE].

[38] C. Csáki, J. Hubisz, S. Lombardo and J. Terning, Gluon vs. photon production of a 750 GeV diphoton resonance, arXiv: 1601.00638 [INSPIRE].

[39] D.B. Kaplan, Flavor at SSC energies: a new mechanism for dynamically generated fermion masses, Nucl. Phys. B 365 (1991) 259 [InSPIRE].

[40] N. Arkani-Hamed, M. Porrati and L. Randall, Holography and phenomenology, JHEP 08 (2001) 017 [hep-th/0012148] [INSPIRE].

[41] R. Contino and A. Pomarol, Holography for fermions, JHEP 11 (2004) 058 [hep-th/0406257] [INSPIRE].

[42] K. Agashe, R. Contino and A. Pomarol, The minimal composite Higgs model, Nucl. Phys. B 719 (2005) 165 [hep-ph/0412089] [INSPIRE].

[43] G.F. Giudice, C. Grojean, A. Pomarol and R. Rattazzi, The strongly-interacting light Higgs, JHEP 06 (2007) 045 [hep-ph/0703164] [INSPIRE].

[44] I. Low, R. Rattazzi and A. Vichi, Theoretical constraints on the Higgs effective couplings, JHEP 04 (2010) 126 [arXiv:0907.5413] [INSPIRE].

[45] E. Perazzi, G. Ridolfi and F. Zwirner, Signatures of massive sgoldstinos at $e^{+} e^{-}$colliders, Nucl. Phys. B 574 (2000) 3 [hep-ph/0001025] [InSPIRE].

[46] D.S. Gorbunov and N.V. Krasnikov, Prospects for sgoldstino search at the LHC, JHEP 07 (2002) 043 [hep-ph/0203078] [INSPIRE].

[47] A. Brignole, J.A. Casas, J.R. Espinosa and I. Navarro, Low scale supersymmetry breaking: effective description, electroweak breaking and phenomenology, Nucl. Phys. B 666 (2003) 105 [hep-ph/0301121] [INSPIRE].

[48] C. Petersson and A. Romagnoni, The MSSM Higgs sector with a dynamical goldstino supermultiplet, JHEP 02 (2012) 142 [arXiv:1111.3368] [INSPIRE].

[49] B. Bellazzini, C. Petersson and R. Torre, Photophilic Higgs from sgoldstino mixing, Phys. Rev. D 86 (2012) 033016 [arXiv:1207.0803] [InSPIRE].

[50] E. Dudas, C. Petersson and P. Tziveloglou, Low Scale Supersymmetry Breaking and its LHC Signatures, Nucl. Phys. B 870 (2013) 353 [arXiv:1211.5609] [INSPIRE].

[51] E. Dudas, C. Petersson and R. Torre, Collider signatures of low scale supersymmetry breaking: a Snowmass 2013 white paper, arXiv:1309.1179 [INSPIRE].

[52] C. Petersson and R. Torre, ATLAS diboson excess from low scale supersymmetry breaking, JHEP 01 (2016) 099 [arXiv: 1508.05632] [inSPIRE].

[53] P. Fayet, Mixing between gravitational and weak interactions through the massive gravitino, Phys. Lett. B 70 (1977) 461 [INSPIRE]. 
[54] P. Fayet, Weak interactions of a light gravitino: a lower limit on the gravitino mass from the decay $\psi \rightarrow$ gravitino anti-photino, Phys. Lett. B 84 (1979) 421 [InSPIRE].

[55] P. Fayet, Scattering cross-sections of the photino and the goldstino (gravitino) on matter, Phys. Lett. B 86 (1979) 272 [INSPIRE].

[56] R. Casalbuoni, S. De Curtis, D. Dominici, F. Feruglio and R. Gatto, A gravitino-goldstino high-energy equivalence theorem, Phys. Lett. B 215 (1988) 313 [INSPIRE].

[57] R. Casalbuoni, S. De Curtis, D. Dominici, F. Feruglio and R. Gatto, High-energy equivalence theorem in spontaneously broken supergravity, Phys. Rev. D 39 (1989) 2281 [INSPIRE].

[58] CMS collaboration, Search for dark matter, extra dimensions and unparticles in monojet events in proton-proton collisions at $\sqrt{s}=8 \mathrm{TeV}$, Eur. Phys. J. C 75 (2015) 235 [arXiv: 1408.3583] [INSPIRE].

[59] C. Csáki, J. Hubisz and S.J. Lee, Radion phenomenology in realistic warped space models, Phys. Rev. D 76 (2007) 125015 [arXiv:0705.3844] [inSPIRE].

[60] W.D. Goldberger, B. Grinstein and W. Skiba, Distinguishing the Higgs boson from the dilaton at the Large Hadron Collider, Phys. Rev. Lett. 100 (2008) 111802 [arXiv:0708.1463] [INSPIRE].

[61] L. Vecchi, Phenomenology of a light scalar: the dilaton, Phys. Rev. D 82 (2010) 076009 [arXiv: 1002.1721] [INSPIRE].

[62] V. Barger, M. Ishida and W.-Y. Keung, Dilaton at the LHC, Phys. Rev. D 85 (2012) 015024 [arXiv: 1111.2580] [INSPIRE].

[63] Z. Chacko, R. Franceschini and R.K. Mishra, Resonance at 125 GeV: Higgs or dilaton/radion?, JHEP 04 (2013) 015 [arXiv: 1209.3259] [INSPIRE].

[64] Z. Chacko and R.K. Mishra, Effective theory of a light dilaton, Phys. Rev. D 87 (2013) 115006 [arXiv: 1209.3022] [INSPIRE].

[65] B. Bellazzini, C. Csáki, J. Hubisz, J. Serra and J. Terning, A Higgslike dilaton, Eur. Phys. J. C 73 (2013) 2333 [arXiv:1209.3299] [InSPIRE].

[66] P. Cox, A.D. Medina, T.S. Ray and A. Spray, Radion/dilaton-Higgs mixing phenomenology in light of the LHC, JHEP 02 (2014) 032 [arXiv:1311.3663] [INSPIRE].

[67] Z. Chacko, R.K. Mishra, D. Stolarski and C.B. Verhaaren, Interactions of a stabilized radion and duality, Phys. Rev. D 92 (2015) 056004 [arXiv:1411.3758] [INSPIRE].

[68] R. Rattazzi and A. Zaffaroni, Comments on the holographic picture of the Randall-Sundrum model, JHEP 04 (2001) 021 [hep-th/0012248] [INSPIRE].

[69] G.F. Giudice, R. Rattazzi and J.D. Wells, Graviscalars from higher dimensional metrics and curvature Higgs mixing, Nucl. Phys. B 595 (2001) 250 [hep-ph/0002178] [INSPIRE].

[70] C. Csáki, M.L. Graesser and G.D. Kribs, Radion dynamics and electroweak physics, Phys. Rev. D 63 (2001) 065002 [hep-th/0008151] [INSPIRE].

[71] B. Bellazzini, C. Csáki, J. Hubisz, J. Serra and J. Terning, A naturally light dilaton and a small cosmological constant, Eur. Phys. J. C 74 (2014) 2790 [arXiv:1305.3919] [InSPIRE].

[72] F. Coradeschi, P. Lodone, D. Pappadopulo, R. Rattazzi and L. Vitale, A naturally light dilaton, JHEP 11 (2013) 057 [arXiv: 1306.4601] [INSPIRE].

[73] E. Megias and O. Pujolàs, Naturally light dilatons from nearly marginal deformations, JHEP 08 (2014) 081 [arXiv: 1401.4998] [INSPIRE]. 
[74] P. Cox and T. Gherghetta, A soft-wall dilaton, JHEP 02 (2015) 006 [arXiv:1411.1732] [INSPIRE].

[75] K.F. Cleary and J. Terning, Marginal breaking of conformal SUSY QCD, arXiv: 1510.08065 [INSPIRE].

[76] K. Harigaya and Y. Nomura, Composite models for the $750 \mathrm{GeV}$ diphoton excess, Phys. Lett. B 754 (2016) 151 [arXiv: 1512.04850] [INSPIRE].

[77] Y. Mambrini, G. Arcadi and A. Djouadi, The LHC diphoton resonance and dark matter, Phys. Lett. B 755 (2016) 426 [arXiv:1512.04913] [InSPIRE].

[78] M. Backovic, A. Mariotti and D. Redigolo, Di-photon excess illuminates dark matter, JHEP 03 (2016) 157 [arXiv: 1512.04917] [INSPIRE].

[79] A. Angelescu, A. Djouadi and G. Moreau, Scenarii for interpretations of the LHC diphoton excess: two Higgs doublets and vector-like quarks and leptons, Phys. Lett. B 756 (2016) 126 [arXiv: 1512.04921] [INSPIRE].

[80] Y. Nakai, R. Sato and K. Tobioka, Footprints of new strong dynamics via anomaly, arXiv: 1512.04924 [INSPIRE].

[81] S. Knapen, T. Melia, M. Papucci and K. Zurek, Rays of light from the LHC, arXiv: 1512.04928 [INSPIRE].

[82] D. Buttazzo, A. Greljo and D. Marzocca, Knocking on new physics' door with a scalar resonance, Eur. Phys. J. C 76 (2016) 116 [arXiv:1512.04929] [INSPIRE].

[83] A. Pilaftsis, Diphoton signatures from heavy axion decays at the CERN Large Hadron Collider, Phys. Rev. D 93 (2016) 015017 [arXiv:1512.04931] [INSPIRE].

[84] R. Franceschini et al., What is the $\gamma \gamma$ resonance at $750 \mathrm{GeV}$ ?, JHEP 03 (2016) 144 [arXiv:1512.04933] [INSPIRE].

[85] S. Di Chiara, L. Marzola and M. Raidal, First interpretation of the $750 \mathrm{GeV}$ di-photon resonance at the LHC, arXiv:1512.04939 [INSPIRE].

[86] T. Higaki, K.S. Jeong, N. Kitajima and F. Takahashi, The QCD axion from aligned axions and diphoton excess, Phys. Lett. B 755 (2016) 13 [arXiv:1512.05295] [INSPIRE].

[87] S.D. McDermott, P. Meade and H. Ramani, Singlet scalar resonances and the diphoton excess, Phys. Lett. B 755 (2016) 353 [arXiv:1512.05326] [INSPIRE].

[88] J. Ellis, S.A.R. Ellis, J. Quevillon, V. Sanz and T. You, On the interpretation of a possible $\sim 750 \mathrm{GeV}$ particle decaying into $\gamma \gamma$, JHEP 03 (2016) 176 [arXiv: 1512.05327] [INSPIRE].

[89] M. Low, A. Tesi and L.-T. Wang, A pseudoscalar decaying to photon pairs in the early LHC Run 2 data, JHEP 03 (2016) 108 [arXiv: 1512.05328] [INSPIRE].

[90] B. Bellazzini, R. Franceschini, F. Sala and J. Serra, Goldstones in diphotons, arXiv: 1512.05330 [INSPIRE].

[91] R.S. Gupta, S. Jäger, Y. Kats, G. Perez and E. Stamou, Interpreting a 750 GeV diphoton resonance, arXiv: 1512.05332 [INSPIRE].

[92] C. Petersson and R. Torre, The $750 \mathrm{GeV}$ diphoton excess from the goldstino superpartner, arXiv: 1512.05333 [INSPIRE].

[93] E. Molinaro, F. Sannino and N. Vignaroli, Minimal composite dynamics versus axion origin of the diphoton excess, arXiv:1512.05334 [INSPIRE]. 
[94] P. Agrawal, J. Fan, B. Heidenreich, M. Reece and M. Strassler, Experimental considerations motivated by the diphoton excess at the LHC, arXiv:1512.05775 [INSPIRE].

[95] A. Ahmed, B.M. Dillon, B. Grzadkowski, J.F. Gunion and Y. Jiang, Higgs-radion interpretation of $750 \mathrm{GeV}$ di-photon excess at the LHC, arXiv:1512.05771 [INSPIRE].

[96] D. Aloni, K. Blum, A. Dery, A. Efrati and Y. Nir, On a possible large width $750 \mathrm{GeV}$ diphoton resonance at ATLAS and CMS, arXiv:1512.05778 [INSPIRE].

[97] Y. Bai, J. Berger and R. Lu, A 750 GeV dark pion: cousin of a dark G-parity-odd WIMP, arXiv: 1512.05779 [INSPIRE].

[98] D. Becirevic, E. Bertuzzo, O. Sumensari and R.Z. Funchal, Can the new resonance at LHC be a CP-odd Higgs boson?, arXiv:1512.05623 [INSPIRE].

[99] L. Bian, N. Chen, D. Liu and J. Shu, A hidden confining world on the $750 \mathrm{GeV}$ diphoton excess, arXiv: 1512.05759 [INSPIRE].

[100] Q.-H. Cao, Y. Liu, K.-P. Xie, B. Yan and D.-M. Zhang, A boost test of anomalous diphoton resonance at the $L H C$, arXiv:1512.05542 [INSPIRE].

[101] J. Chakrabortty, A. Choudhury, P. Ghosh, S. Mondal and T. Srivastava, Di-photon resonance around $750 \mathrm{GeV}$ : shedding light on the theory underneath, arXiv:1512.05767 [INSPIRE].

[102] W. Chao, R. Huo and J.-H. Yu, The minimal scalar-stealth top interpretation of the diphoton excess, arXiv:1512.05738 [INSPIRE].

[103] P. Cox, A.D. Medina, T.S. Ray and A. Spray, Diphoton excess at $750 \mathrm{GeV}$ from a radion in the bulk-Higgs scenario, arXiv:1512.05618 [INSPIRE].

[104] C. Csáki, J. Hubisz and J. Terning, Minimal model of a diphoton resonance: Production without gluon couplings, Phys. Rev. D 93 (2016) 035002 [arXiv:1512.05776] [INSPIRE].

[105] D. Curtin and C.B. Verhaaren, Quirky explanations for the diphoton excess, Phys. Rev. D 93 (2016) 055011 [arXiv: 1512.05753] [INSPIRE].

[106] S.V. Demidov and D.S. Gorbunov, On sgoldstino interpretation of the diphoton excess, arXiv: 1512.05723 [INSPIRE].

[107] B. Dutta, Y. Gao, T. Ghosh, I. Gogoladze and T. Li, Interpretation of the diphoton excess at CMS and ATLAS, Phys. Rev. D 93 (2016) 055032 [arXiv:1512.05439] [InSPIRE].

[108] A. Falkowski, O. Slone and T. Volansky, Phenomenology of a $750 \mathrm{GeV}$ singlet, JHEP 02 (2016) 152 [arXiv: 1512.05777] [INSPIRE].

[109] A. Kobakhidze, F. Wang, L. Wu, J.M. Yang and M. Zhang, 750 GeV diphoton resonance in a top and bottom seesaw model, arXiv:1512.05585 [INSPIRE].

[110] R. Martínez, F. Ochoa and C.F. Sierra, Diphoton decay for a $750 \mathrm{GeV}$ scalar boson in an $\mathrm{U}(1)^{\prime}$ model, arXiv:1512.05617 [INSPIRE].

[111] S. Matsuzaki and K. Yamawaki, $750 \mathrm{GeV}$ diphoton signal from one-family walking technipion, arXiv:1512.05564 [INSPIRE].

[112] J.M. No, V. Sanz and J. Setford, See-saw composite higgses at the LHC: linking naturalness to the $750 \mathrm{GeV}$ di-photon resonance, arXiv:1512.05700 [INSPIRE]. 